\title{
The recent 500th anniversary of Leonardo da Vinci's death: a reminder of his contribution in the field of fluid mechanics
}

\author{
Michele Mossa ${ }^{1}$ iD
}

Received: 24 March 2020 / Accepted: 23 May 2020 / Published online: 29 May 2020

(c) Springer Nature B.V. 2020

\begin{abstract}
In this work, a summary of Leonardo da Vinci's contribution in the field of fluid mechanics is reported. Some theoretical laws or fluid flows analyzed or drawn by Leonardo are highlighted, such as vortices, flow fields with obstacles, the continuity law, waves, velocity profiles in open channels, as well as some ingenious contributions in the field of hydraulic constructions and machines.
\end{abstract}

Keywords Leonardo da Vinci · 500th Anniversary of Leonardo da Vinci's death · History of fluid mechanics

\section{Introduction}

The history of continuum mechanics can be traced from the early work of the Hellenic period up to the present century. This history is based upon early work in statics, deformable solids, dynamics, fluid mechanics and aerodynamics. The unifying theory of continuum mechanics came in the 1900s combined with the advances in thermodynamics and rheology.

It is believed that one of the precursors of the continuum mechanics and studies of the interactions between solids and fluids was Leonardo da Vinci (1452-1519), whose 500th anniversary of his death was celebrated in 2019. Leonardo was ahead of his time. However, in some respects, crucially, he was obviously a man of his time. It is correct to say that the innovations proposed by Leonardo highlight his ability to operate better than his predecessors or contemporaries in the scientific context of his time [1,2].

Among the wide range of phenomena that Leonardo analyzed and represented, the behavior of liquids must be considered. In his Codex Leicester, Leonardo da Vinci [3] highlights his revolutionary ideas on fluid dynamics. The numerous drawings contained in the codes prove that Leonardo's interest in fluid mechanics works and water is only one aspect of his method of observation and representation of the landscape, all at once, artistic, naturalistic and related to engineering.

Michele Mossa

michele.mossa@poliba.it

1 Department of Civil, Environmental, Land, Building Engineering and Chemistry, Polytechnic University of Bari, Via E. Orabona 4, 70125 Bari, Italy 
Some examples of hydraulic phenomena or hydraulic works, analyzed or drawn by Leonardo, will be shown.

It should be noted that the present paper shows only a minimal part of Leonardo's studies on water. In fact, for the sake of brevity, it was necessary to make choices, cutting, unfortunately, numerous other contributions of Leonardo.

\section{Vortices}

Although Leonardo da Vinci was not the first to advocate the experimental method, which is accredited to Galieo Galiei, he differed considerably from others in the degree to which he exemplified what he advocated. His basic premise was "remember when discoursing on the flow of water to adduce first experience and then reason".

Some phenomena that he was the first to sketch or describe are the formation of eddies at abrupt expansions (Fig. 1) and the streamlines in vortices.

Leonardo was fascinated by the vortices, not only of water, but also by those, governed by the same laws, of air and blood in the ventricles and in the atria of the heart. In the Codex Leicester he provides depictions of vortices and their infinite variability which are unprecedented and will not have a long following. With its devastating spiral dynamics, the vortex is an expression of the force of nature. Nothing can resist its demolition action that continually changes the surface and belly of the Earth.

Based on observations of nature, Leonardo identified three types of vortices, namely those level with the surface, those with the center raised (i.e., vortices present in turbulent zones of air) and those whose center tends downwards (i.e., swirls in a sink). During his time, Leonardo did not have the mathematical tools for an analytical development of the vortex theory, which would have been developed hundreds of years later, starting from Helmholtz [4]. Nevertheless, Leonardo's drawings of the eddies present in river and lake flows, around obstacles or near the confluences of rivers are extremely detailed and of great interest. The drawings of vortices present in the Codex Leicester are linked to others, such as those of the royal collection of Windsor Castle, particularly important (see Fig. 1).

To understand how in many cases Leonardo's intuitions and observations are still current, think of the vortex structure of a hydraulic jump. Only many years later after Leonardo, Guglielmini [5] and Bidone [6] notice the phenomenon of a hydraulic jump.

Later, Bélanger [7] obtained the well-known equation of hydraulic jumps (see Leutheusser and Alemu [8], that considers the head loss of the flow and the dynamic similarity [9].

In this fascinating story of hydraulic jumps, which is still a very current topic (see, for example, Gualtieri and Chanson [10], Ben Meftah et al. [11, 12], De Padova et al. [13], among many recent papers), Leonardo intervened long before Guglielmini himself, not to solve the equation describing the phenomenon, which, as already written, happened much later, but to observe and draw the vortices in the flow in great detail. 


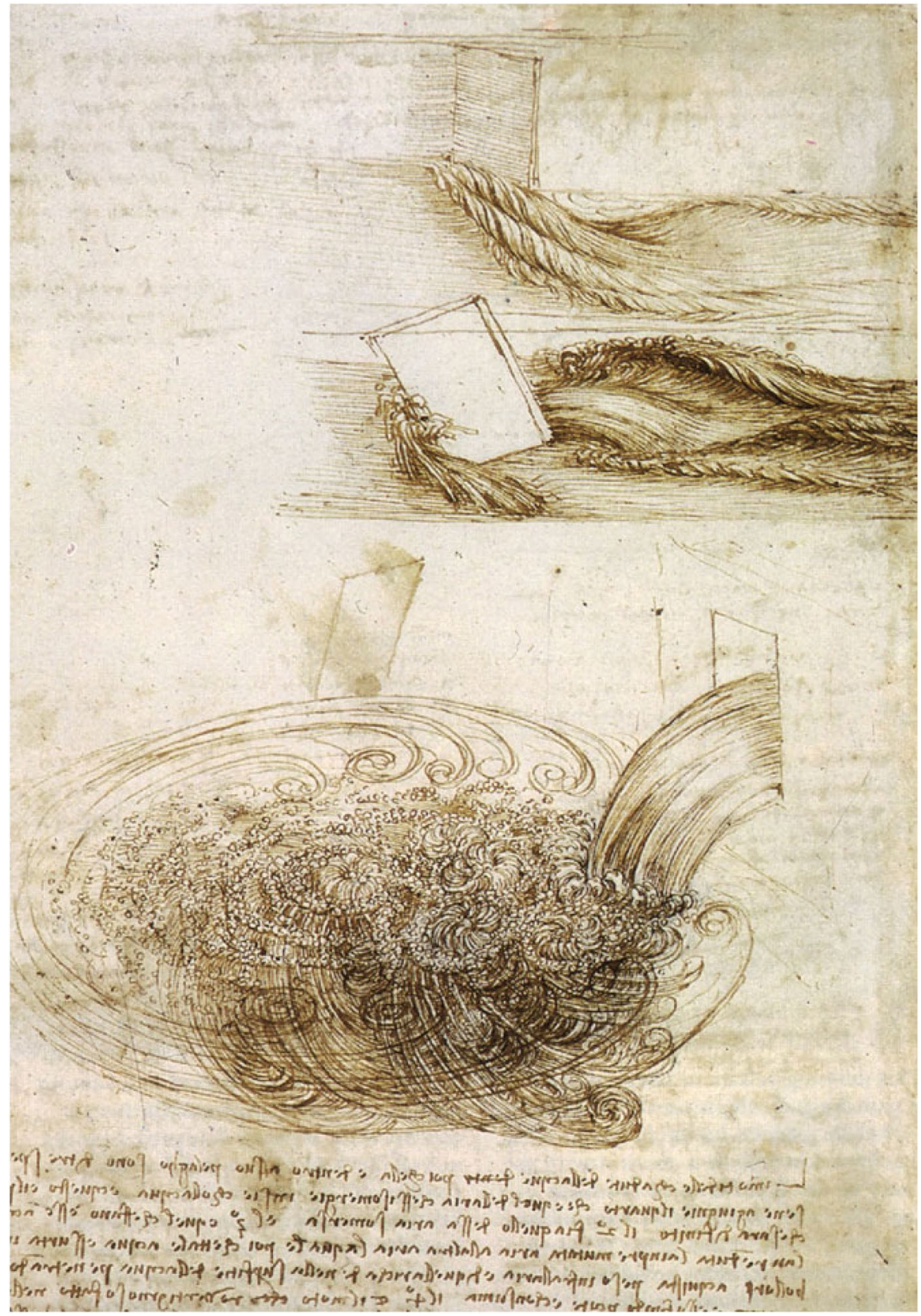

Fig. 1 Vortex studies in currents (Windsor Castle, The Royal Collection, RL 1266ov)

\section{Studies on motion fields in the presence of obstacles}

The interest of the most innovative research of the interaction between flows and vegetation $[14,15]$ is linked, for example, to the effects that mangroves and, more generally, coastal 
plants have in terms of attenuation of waves due to large storms or tsunamis. Furthermore, the coastal vegetation could naturally improve the beach stability $[16,17]$.

Leonardo was a precursor also in this field, at least in terms of observation and graphic reproduction of the phenomenon. In this regard, see Fig. 2, which represents an elderly man sitting on a rock intending to observe a current (The Royal Collection, RL 12579). In that table the objects or obstacles, synonyms that Leonardo alternate in the numerous papers of his code, are the barriers, natural or artificial, situated in the riverbed, which make a "firm shield to the event of the waters".

Leonardo wrote about the effects on a channel flow caused by these obstacles, based on their shape, number and mutual distance. In this regard, particularly interesting is also the table shown in Fig. 3.

\section{The continuity law}

Qualitative ideas on the concept known today as the continuity law, in the sense of conserved mass used by the French chemist Antoine Lavoisier (1743-1794), had already been formulated by the ancient atomists. Seneca quotes Democritus, who compared the corpuscles of air to a crowd lining a narrow passage. In f. 57v of Ms. A Leonardo wrote: "If there is a place that has 3 different widths [...] and the first smaller width enters the second 4 times and the second comes twice in the third, I say that the little men who will fill [...] the said places [...], that when the men of the greater place will make 1 step, those of the second smaller width will do 2, and those of the third place [...] in that the same time in all the movements, which pass through various widths of places." See Fig. 4.

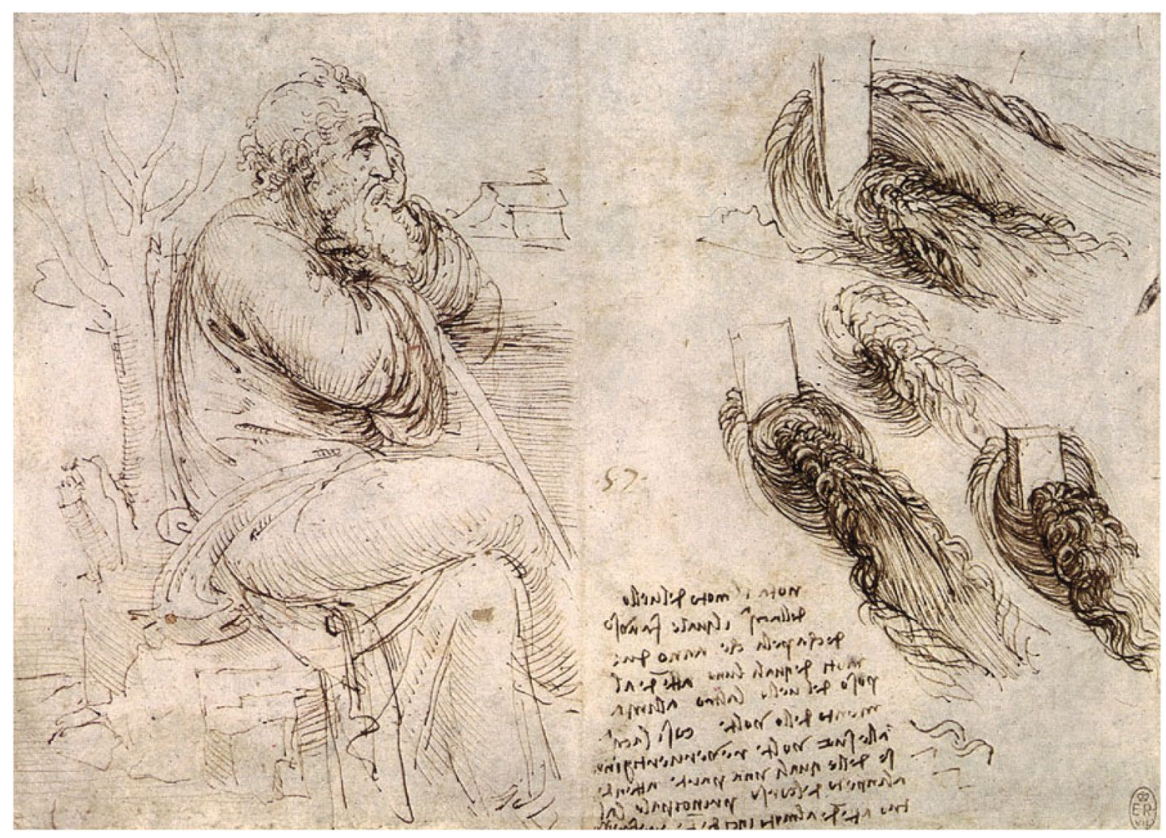

Fig. 2 Seated old man, studying a water current (The Royal Collection, RL 12579) 


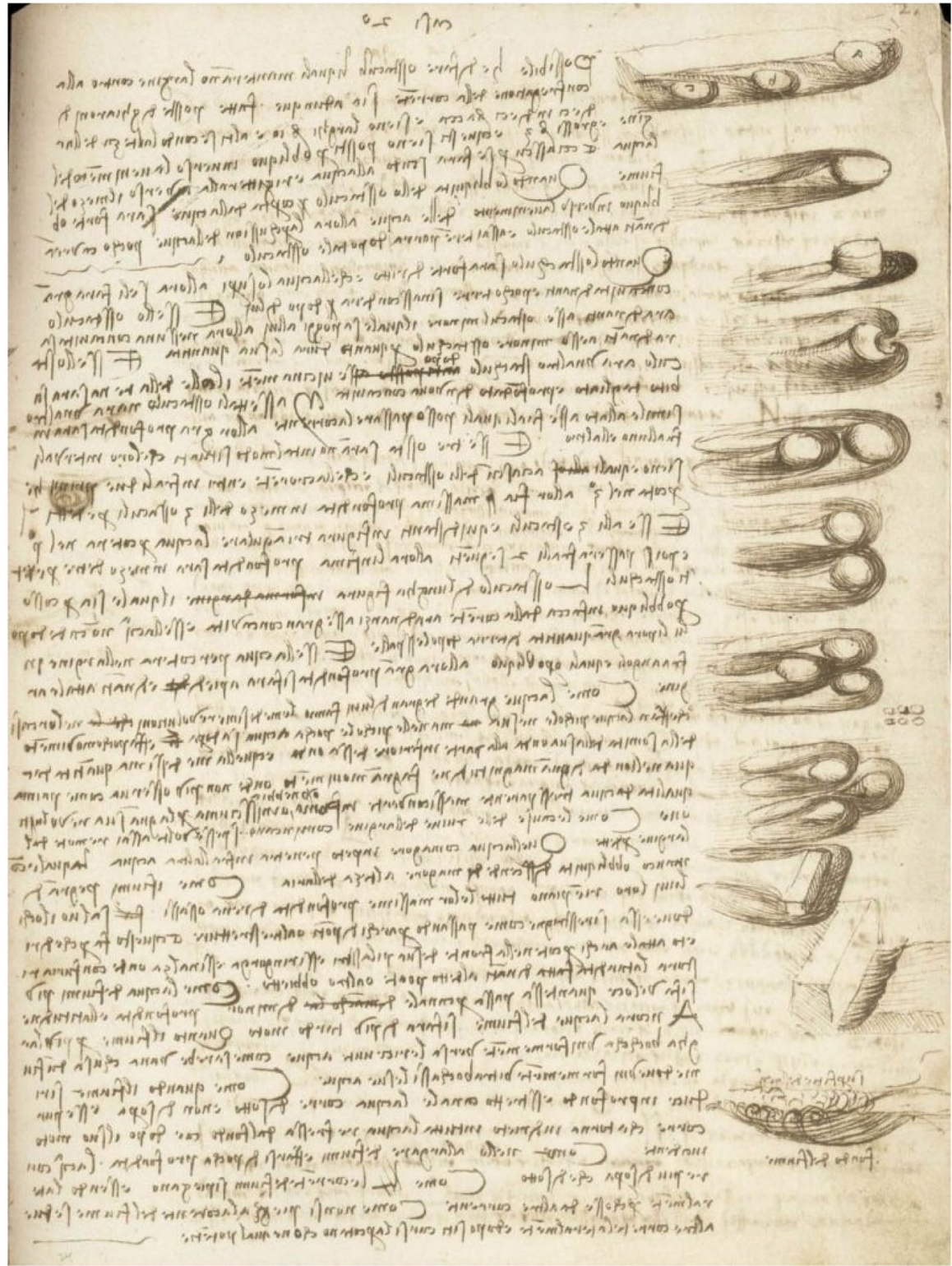

Fig. 3 Interaction between water, riverbed, and obstacles. Hydraulic engineering (Leicester Codex, 24r)

\section{Waves and combination of art and science}

Leonardo's observation on the absence of mass transport in waves is also memorable, with his famous example of the waves generated by the wind in a wheat field. As shown by Capra [18] Leonardo observed that "Accade sovente che l'onda si allontani dal suo punto di creazione, mentre l'acqua non si muove, come le onde create dal vento in un campo di 
Fig. 4 Leonardo da Vinci: "[...] and this portion you will find in all the movements that pass through various widths of places". The equation of continuity explained with the example of a group of marchers forced to pass through two bottlenecks, Ms. A. f. $57 \mathrm{v}$, detail

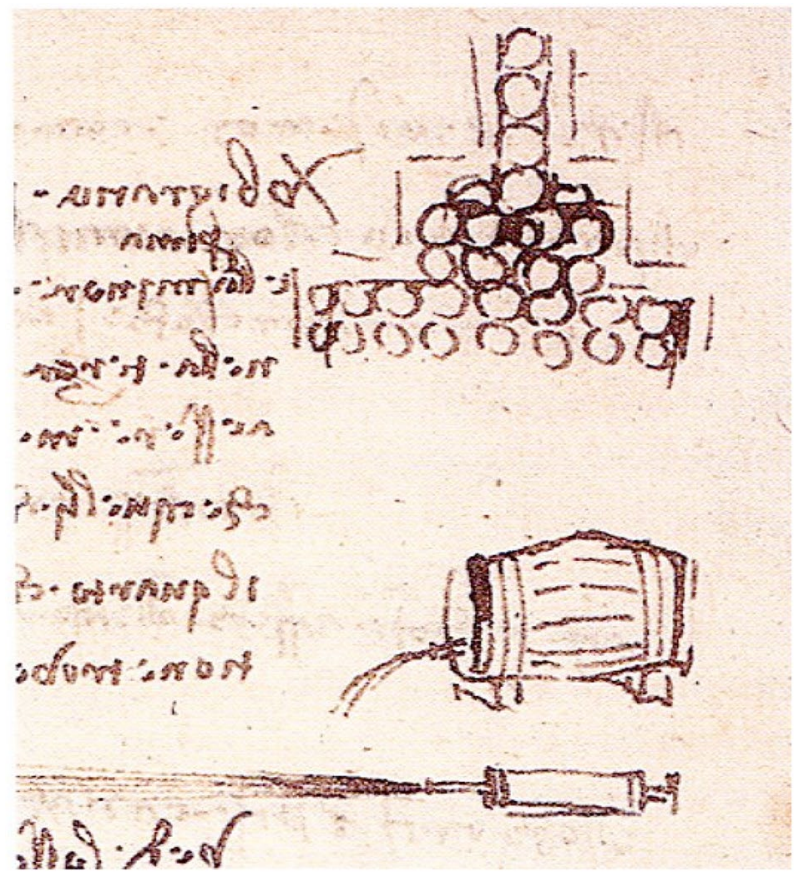

grano, dove vediamo le onde correre attraverso il campo mentre il grano rimane al suo posto" (i.e. "The wave flees from the place of its creation without the water changing its position, like the waves which the course of the wind makes in wheat fields, where one sees the waves running over the field without the wheat field changing its place").

Leonardo drew the waves several times and an example is shown in Fig. 5.

Leonardo da Vinci's intuitions are often linked to his art form. For example, it is possible to believe that Leonardo interpreted the waves as a universal form of propagation of emotions, not necessarily linked to the transfer of the mass. From this point of view, it is believed that his famous painting "The Last Supper" also represents a wave of emotions, which, accompanied by silence, is born with the words of Jesus who predicts his betrayal. This is a typical example of the wonderful combination of art and science, often present

Fig. 5 Leonardo's sketch of a wave (Codex Leicester, 4v)

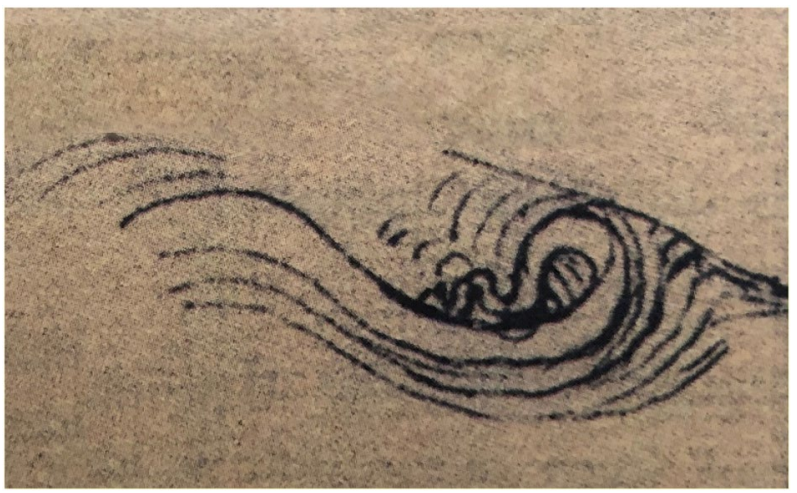


in Leonardo's work. The many drawings in Leonardo's codices prove how his interest for hydraulic works and for water were but a single aspect of his method of observation and representation of landscapes; at the same time, this representation made use of points of view that were equally artistic, naturalistic, and engineering.

\section{Qualitative study of the velocity profile in open channels}

As shown by Di Fidio and Gandolfi [19], Leonardo had a thorough understanding of the velocity distribution in rivers. He noted that the water motion resistance, which is greater close to the riverbed, is responsible for smaller velocities at the bottom. For the same resistance, the velocities diminish from the river's central axis towards its banks. Leonardo carried out some experiments on the velocity variations along a vertical direction in a river, inventing a new floating device (see Fig. 6 from Cardinali [20], composed of a rod, whose upper part is inserted in a floating leather bag, and the lower part in a stone. If the rod leans upstream, the water current velocities are greater in the lower part of the rod, and vice versa if the rod leans downstream. Of course, if the rod remains vertical, the velocity current is constant along the rod length.

\section{Remediation works, hydraulic machines and locks}

The marsh reclamation works to which Leonardo made his contribution were also numerous and often decisive. As an example, we will briefly describe the reclamation of the Pontine Marshes, a malaria-infested area of about $800 \mathrm{~km}^{2}$ south-west Rome, in the territory of the Papal State. In 1514 Giovanni de' Medici, Pope Leo X, granted to his cousin, Julius de' Medici, later Pope Clement VII, a concession for the work of drainage, which resulted in the cutting of a canal named Fiume Giuliano after the pope's brother, Giuliano de' Medici, whose service Leonardo da Vinci was in at the time. Probably during this time Leonardo prepared a map showing his scheme of drainage, in which he proposed to recut the ancient Roman canal running parallel to the Appian Way, and, as previously done by the Romans, use it as a cut-off channel for the waters coming down from the Lepini Mountains. For further details, see Ciriacono [21].

Fig. 6 Rod floating device used by Leonardo to measure relative speed between two layers

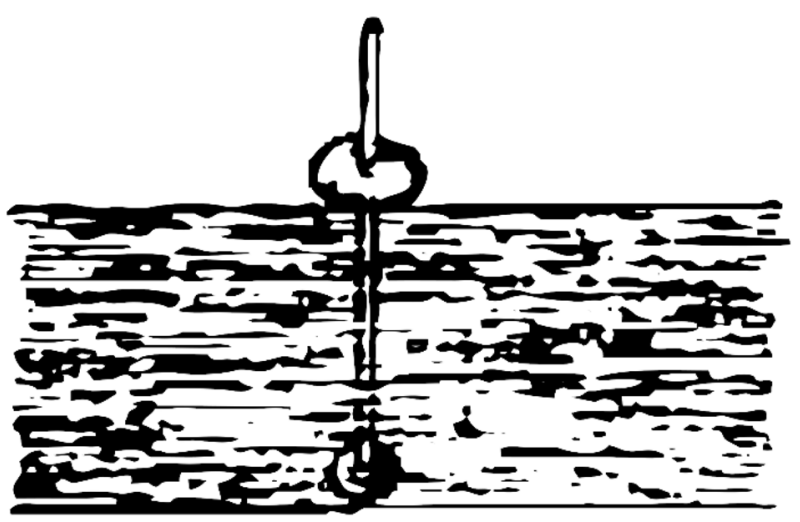


Like Archimedes, Leonardo made great contributions in hydraulic works and machines. Leonardo da Vinci repeatedly expressed admiration for Archimedes during his lifetime. Not only was he fascinated by the legend of Archimedes as inventor of prodigious war machines, but also by his successful approach to problems of statics and engineering, from which practical applications could be drawn. Particularly, Leonardo showed similar admiration in the statics field, studied the treatise "On Plane Equilibria", even referring to it on the cover of Manuscript F ("Archimedes, de centro gravitatis").

Also the water screw, popularly known as the Archimedes' screw, the well-known machine used for transferring water from a low-lying body of water into irrigation ditches (commonly attributed to Archimedes, even though some historical documents show that it was used in Ancient Egypt) was rediscovered and reanalyzed by Leonardo.

As an example of hydraulic works, Leonardo's contribution in Milan will be briefly describe.

Milan was like Venice, with its navigable canals, an effective and efficient network of communication. In just 35 years, from 1439 to $1475,90 \mathrm{~km}$ of canals were built in the Milan area. A record that no other city could have boasted.

Leonardo da Vinci arrived in Milan in 1482 and was commissioned by Ludovico il Moro to make canals navigable. See Fig. 7 for an example of locks proposed by Leonardo.

\section{Conclusions}

In one of his aphorisms, Leonardo da Vinci observed that "Mechanics is the paradise of mathematical sciences, because with that one comes to the mathematical fruit". Leonardo was a pioneer of modern science, however, in some respects, he was also a man of his time. Its versatility was prefigured by the great artist-engineers of the Italian Renaissance. In his work on physics, Leonardo was heir to medieval theories of statics and dynamics, often linked to his art form.

Five hundred years after his death, in this note some flow fields and hydraulic laws analyzed or only simply drawn by Leonardo have been highlighted, such as vortices, flow fields with obstacles, the continuity law, waves, velocity profiles in open channels, as well as some ingenious contributions in the field of hydraulic constructions and machines. 


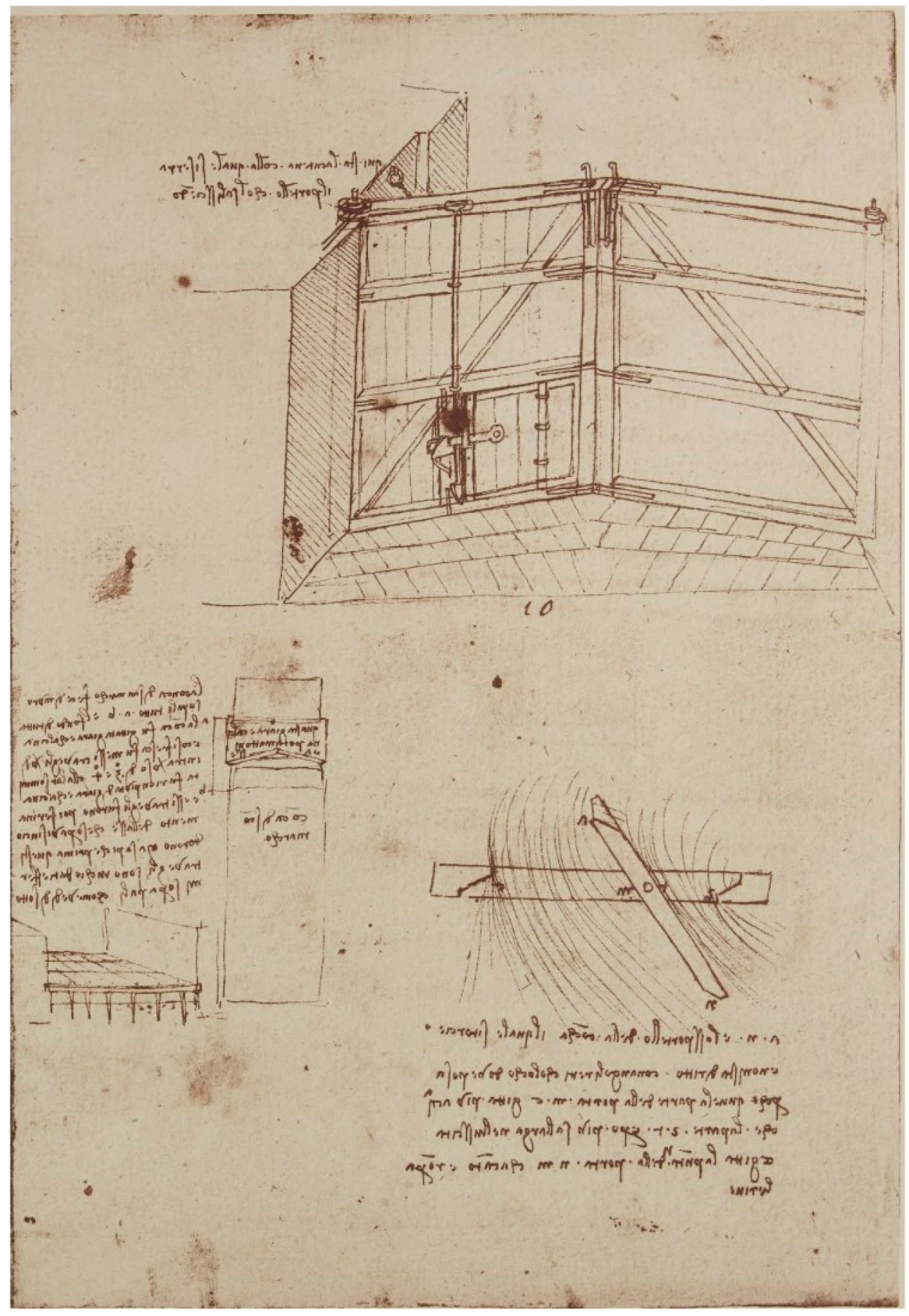

Fig. 7 Lock at San Marco, from Codex Atlanticus, f. 240r-c (656r-a new numbering)

\section{References}

1. Kemp M (2019) Leonardo's laboratory: studies in flow. Nature 571:322-323 
2. McCurdy E (1920) Leonardo da Vinci. Nature 105(2637):340-342

3. Leonardo da Vinci (1510) Codice Leicester, Bill Gates's private collection

4. Helmholtz H (1858) Über Integrale der hydrodynamischen Gleichungen, welche den Wirbelbewegungen entsprechen. J Die Reine Angew Math 55, ISSN 0075-4102

5. Guglielmini D (1739) Della natura de' fiumi, Nuova Edizione con le annotazioni di Eustachio Manfredi, Bologna

6. Bidone G (1820) Expériences sur la propagation des remous. Mem della Reale Accad delle Sci Torino 30:195-292

7. Bélanger JB (1828) Essai sur la Solution Numérique de quelques Problèmes Relatifs au Mouvement Permanent des Eaux Courantes (Essay on the Numerical Solution of Some Problems relative to Steady Flow of Water). Carilian-Goeury, Paris

8. Leutheusser HJ, Alemu S (1978) Flow separation under hydraulic jump. J Hydraul Res IAHR 17(3):193-206

9. Bakhmeteff BA, Matzke AE (1936) The hydraulic jump in terms of dynamic similarity. ASCE Trans 101, paper no. $1935,630-680$

10. Gualtieri C, Chanson H (2007) Experimental analysis of Froude number effect on air entrainment in the hydraulic jump. Environ Fluid Mech 7:217-238

11. Ben Meftah M, De Serio F, Mossa M, Pollio A (2007) Analysis of the velocity field in a large rectangular channel with lateral shockwave. Environ Fluid Mech 7(6):519-536

12. Ben Meftah M, Mossa M, Pollio A (2010) Considerations on shock wave/boundary layer interaction in undular hydraulic jumps in horizontal channels with a very high aspect ratio. Eur J Mech B/Fluids 29(6):415-429

13. De Padova D, Mossa M, Sibilla S, Torti E (2013) 3D SPH modelling of hydraulic jump in a very large channel. J Hydraul Res 51(2):158-173

14. Mossa M, De Serio F (2016) Rethinking the process of detrainment: jets in obstructed natural flows. Sci Rep Nat 6, Article number 39103, ISSN: 20452322. https://doi.org/10.1038/srep39103

15. Mossa M, Ben Meftah M, De Serio F, Nepf HM (2017) How vegetation in flows modifies the turbulent mixing and spreading of jets. Sci Rep 7(1), Article number 6587. https://doi.org/10.1038/s41598-01705881-1

16. Tognin D, Peruzzo P, De Serio F, Ben Meftah M, Carniello L, Defina A, Mossa M (2019) Experimental setup and measuring system to study solitary wave interaction with rigid emergent vegetation. Sensors 19(8):1787. https://doi.org/10.3390/s19081787

17. Peruzzo P, De Serio F, Defina A, Mossa M (2018) Wave height attenuation and flow resistance due to emergent or near-emergent vegetation. Water 10(4):402. https://doi.org/10.3390/w10040402

18. Capra F (2013) Learning from Leonardo-decoding the notebooks of a genius. Berrett-Koehler Publishers Inc, San Francisco

19. Di Fidio M, Gandolfi C (2017) Flow velocity measurement in Italy between Renaissance and Risorgimento. J Hydraul Res 49(5):578-585. https://doi.org/10.1080/00221686.2011.594599

20. Cardinali F (ed) (1828) Leonardo da Vinci (1828). Del moto e misura dell' acqua di Leonardo da Vinci, Bologna

21. Ciriacono S (ed) (2017) Land drainage and irrigation—studies in the history of civile engineering, vol 3. Routledge, Abingdon

Publisher's Note Springer Nature remains neutral with regard to jurisdictional claims in published maps and institutional affiliations. 